\section{$\underset{\substack{\text { hommes } \\ \text { \& migrations }}}{ }$}

\section{Hommes \& migrations}

Revue française de référence sur les dynamiques

migratoires

$1295 \mid 2012$

Algérie - France : une communauté de destin

\title{
La tectonique des peuples
}

\section{Mustapha Harzoune}

\section{(2) OpenEdition \\ Journals}

\section{Édition électronique}

URL : http://journals.openedition.org/hommesmigrations/1061

DOI : 10.4000/hommesmigrations.1061

ISSN : 2262-3353

\section{Éditeur}

Musée national de l'histoire de l'immigration

\section{Édition imprimée}

Date de publication : 1 janvier 2012

Pagination : 6-10

ISSN : 1142-852X

Référence électronique

Mustapha Harzoune, «La tectonique des peuples », Hommes \& migrations [En ligne], 1295 | 2012, mis en ligne le 01 janvier 2014, consulté le 03 mai 2019. URL : http://journals.openedition.org/ hommesmigrations/1061; DOI : 10.4000/hommesmigrations.1061 


\section{La tectonique des peuples}

Par Mustapha Harzoune, journaliste

Le cinquantième anniversaire de l'indépendance algérienne offre l'occasion pour notre revue de consacrer deux numéros aux relations franco-algériennes, en privilégiant un pays - mentionné sur aucune carte -, le pays de la migration, et donc, ce que l'on oublie un peu trop dans le débat public actuel, des vies d'hommes et de femmes nourries de rencontres, d'échanges, d'amitiés, de déconvenues parfois, mais aussi d'amours ${ }^{(1)}$. De ce pays invisible et sans drapeaux, né dans les interstices des déraisons de l'Histoire, Hommes $\mho$ Migrations est citoyen. L'un des tout premiers. En 1947, Jacques Ghys, un Père blanc ${ }^{(2)}$, fonde l'association Amana (Assistance morale et aide aux Nord-Africains) qui vise à apporter une aide sociale aux immigrés nord-africains dont le gros des troupes est constitué alors d'Algériens. En 1950, l'association crée deux publications, dont les Cahiers nordafricains. Lobjectif est d'informer des réalités migratoires en France, notamment des conditions de vie des Algériens, et de "jeter des passerelles culturelles entre le nord et le sud de la Méditerranée $e^{(3) "}$. De cette publication pionnière, informée et parfois gênante, est née Hommes ê Migrations qui a contribué à sortir l'immigration et les immigrés, en particulier les immigrés algériens, de la relégation spatiale et conceptuelle, pour les inscrire comme "réalité incontournable" dans le devenir de la société française, participant à plein au "vivre-ensemble" rassemblant Français et immigrés.

C'est dire si les termes et l'esprit de ces deux dossiers allaient de soi : favoriser les éléments prospectifs et humains sur les inventaires (nécessairement présents) et sur certaines approches théoriques (nécessairement mentionnées) qui ont déjà trouvé et trouveront, en cette année prolixe en publications et colloques, à s'exprimer dans d'autres cadres ; partir des deux sociétés et rassembler des chercheurs, universitaires et journalistes, français et algériens, pour interroger cette fameuse "communauté de destin" à l'aune des cinq décennies passées. 


\section{Du XIXe siècle à aujourd'hui, des Algériens dans l'Hexagone}

Il est courant de faire remonter aux premières années du XX $\mathrm{XX}^{\mathrm{e}}$ siècle, en 1907, du côté de Marseille, l'arrivée des premiers immigrés algériens sur le sol de France. Viennent ensuite la mobilisation de la Première Guerre mondiale et surtout, dans l'entredeux-guerres, l'arrivée significative des travailleurs. Parfois, une poignée d'Algériens est mentionnée du côté des savonneries marseillaises à la toute fin du XIX siècle, voire d'autres compatriotes perdus dans le Paris de la Commune. Avant 1910, quelque 9600 Kabyles devaient s'être aventurés en métropole ${ }^{(4)}$.

L'écrivain Abdelkader Djemaï, non sans malice, s'amuse à faire remonter encore plus loin dans le temps l'arrivée des Algériens : 1847 ! Rien moins. Cette année, en effet, voit débarquer l'émir Abd el Kader et le reliquat de sa célèbre smala : une centaine d'hommes, de femmes et d'enfants. En voilà une belle figure d'exilé "en deuil de sa liberté" ! Retenu durant cinq années dans une captivité itinérante qui le conduit de Toulon à Amboise en passant par Pau, Abdelkader resta droit, digne, sans ressentiment pour ses ennemis d'hier. Un vrai chibani des temps modernes. Symbolique fut cette première "trajectoire" algérienne en France : après les épreuves de la prison, il fut trimbalé comme curiosité exotique avant de devenir la coqueluche du Tout-Paris et d'imposer le respect, notamment à Napoléon III, par sa sagesse, son savoir et sa rectitude morale. Homme de religion et mystique, il aurait flirté avec quelques loges maçonniques. Il prôna "la pluralité des mondes" et vit en tout être humain, croyant ou incroyant, un frère - "Tout être est mon être", écrit-il, inaugurant sans doute les bricolages identitaires de demain. Charles X, en lançant son armada sur Alger, ignorait qu'il ouvrait les portes - et les bras - du royaume et demain de la République à l'épopée algérienne en France.

Cette épopée, aux voies souvent impénétrables, commença tôt. En 1994, Mehdi Lallaoui publiait un livre et réalisait un documentaire sur les Kabyles du Pacifique (éditions Au Nom de la mémoire), lointains descendants des révoltés de 1871 déportés en Nouvelle-Calédonie. Dans les cales qui emportaient les malheureux, d'autres déportés partagèrent leur sort : les survivants de la Commune. Une "communauté de destin" liera le devenir des enfants de la terre algérienne et ceux du pavé parisien. Ainsi, Aziz ben Cheikh el Haddad, le principal chef de l'insurrection après el Mokrani, s'est éteint le 22 août 1895 chez Eugène Mourot, compagnon de déportation qui habitait au 45 boulevard de Ménilmontant, face au Père-Lachaise à Paris. Les anciens de la Commune se cotiseront pour le rapatriement du défunt vers son Algérie natale ${ }^{(5)}$. 
De l'émir Abdelkader à ces Algériens de France devenus, depuis deux, trois, quatre générations, français à part entière ; de ce milieu du XIX siècle à ce début de troisième millénaire en passant par la Commune, la Nouvelle-Calédonie, les deux guerres mondiales, les Trente Glorieuses, la Marche des Beurs et autre manifeste "Qui fait la France? (6)", Français et Algériens ont ensemble écrit quelques pages lumineuses, hédonistes, immanentes de l'histoire franco-algérienne. Sans tambour ni trompette. Sans sabre ni goupillon. Sans écouter les bonimenteurs. Simplement en se laissant guider par les hasards de l'existence, les nécessités de l'entraide, le frôlement des peaux et des cceurs. Comme l'écrit Gérard Noiriel à propos des vingtcinq années qui courent de la décennie cinquante au milieu des années soixante-dix : "Le silence a souvent des vertus. En l'occurrence, le fait que les professionnels du discours public se soient désintéressés de l'immigration va permettre aux personnes directement concernées de se fondre progressivement dans la population française

Ces pages heureuses ne recouvrent pas les pages noires et les heures sombres: les 7 Algériens tués par balle et les 44 blessés à Paris du 14 juillet 1953, la guerre d'Algérie et les morts du 17 octobre 1961, les crimes des années soixante-dix - "on peut estimer qu'entre 1971 et 1977 au moins 70 Algériens ont été victimes de crimes à caractère raciste en France ${ }^{(8)}$.' Cela ne fait pas oublier non plus, aujourd'hui, les "bavures", les fragilités sociales, les nombreuses discriminations, la frilosité des élites à laisser quelques strapontins à leurs concitoyens (et concitoyennes !) ou encore les craintes du Haut Conseil à l'intégration" à propos de l'immigration dite "subie" (entendre regroupement familial et "mariages ethniques" entre Français et étrangers de même origine nationale) comme les phénomènes de ghettoïsation, de ségrégation ethnique ou les replis identitaires.

\section{De l'engagement politique des pionniers à la réussite de nouvelles icônes}

Mais voilà ! Ce même $\mathrm{HCI}$, citant les conclusions de l'enquête $\mathrm{TeO}$, rappelle que "65\% des descendants dimmigrés vivent en couple avec des personnes de la population majoritaire". Mais voilà ! Il y a aussi l'histoire, rappelée récemment par le film d'Ismaël Ferroukhi Les Hommes libres ${ }^{(10)}$. Avant, il y eut le documentaire du réalisateur Derri Berkani, Une résistance oubliée, la Mosquée de Paris (1991), et son travail sur la résistance des Francs-tireurs et partisans algériens. Il y eut justement Mohamed Lakhdar, FTP de la première heure, fusillé le 31 janvier 1943. Comme il y eut ce tract retrouvé par le même Derri Berkani, rédigé en kabyle par les FTP algériens, qui, sous le titre "Comme tous nos enfants", lançait cet appel : "Hier, à l'aube, 
les juifs de Paris ont été arrêtés, les vieillards, les femmes comme les enfants, en exil comme nous, ouvriers comme nous, ce sont nos frères et leurs enfants sont nos enfants. Si quelqu'un d'entre vous rencontre un de ces enfants, il doit lui donner asile et protection, le temps que le malheur passe $e^{(11)}$."

Car il y eut la fraternité du monde ouvrier, la solidarité des luttes et des engagements syndicaux, le quotidien des usines et des quartiers populaires ${ }^{(12)}$, les dimanches ensoleillés du populo, l'allégresse de l'anisette bue au zinc et le partage du cassecroûte et du couscous.

Il y eut aussi Élise et Arezki dans Élise ou la vraie vie, le roman de Claire Etcherelli (Denoël, 1967), et le film de Michel Drach (1970). Tassadit Imache et cette Fille sans histoire (Calmann-Levy, 1989) née justement à la croisée de tous les chemins, de toutes les "mises à l'écart", de tous les "dilemmes" du devenir franco-algérien"(13). Il y a l'accent de rocaille et de brique rouge de Magyd Cherfi, dont les mots portent les rages contenues et les amours blessées de plusieurs générations. Il y a sa "compatriote" toulousaine, l'inestimable Juliette, électron libre de la chanson française, rattachée à la branche des Piaf et autre Mouloudji.

Il y a aussi Arezki Idjerouidène qui, d'agent de comptoir dans une agence de voyages, est devenu président du groupe Go Fast et de Aigle Azur, la deuxième compagnie aérienne française derrière Air France. Ou encore Pierre Rabhi, ce "Gandhi à la française (14)", penseur de premier plan d'une "sobriété heureuse" et de l'urgence écologique et humaine de nouveaux modèles économiques. Autant, et combien plus encore, de personnalités, de réussites, de rencontres qui bouleversent les paradigmes et enrichissent la société française à l'image de ces milliers d'anonymes, de cette jeunesse dynamique et "motivée" si active au sein des formations politiques, des associations d'entraide, des associations de quartier et de la moindre lutte citoyenne du pays. La France a changé. Et il n'est pas seulement anecdotique qu'un Zinedine Zidane, lointain héritier d'un Alain Mimoun ou d'un Abdelkader Zaaf, figure, encore en 2011, parmi les personnalités préférées des Français ou que le couscous berbère, de plus en plus républicain, titille la bourgeoise blanquette sur l'échelle gustative de nos concitoyens.

Tout cela s'est fait par "le bas", pour reprendre l'expression d'Alain Tarrius ${ }^{(15)}$. Une tectonique des peuples qui a su, sans le dire et parfois sans le vouloir, bousculer les frontières, les inimitiés et les préjugés pour faire sortir de terre de nouvelles montagnes à escalader. Depuis cinquante ans, et même plus, l'immigration algérienne participe de ce processus. Par deux fois au moins elle imprima deux formidables bifurcations dans l'histoire des hommes et des pays. La première remonte aux années vingt quand, en son sein, commencent à germer les idées de dignité, de liberté et d'indépendance. La seconde, quand les chibanis d'aujourd'hui, au prix d'un double 
sacrifice - celui de l'exil d'abord et celui du retour ensuite -, ont entériné le fait qu'ils finiraient leurs jours ici, en France, proches de leurs enfants. Ce fut le clinamen de Mohamed qui, au lieu de "prendre sa valise", la laissa en haut de l'armoire. Sans le savoir, ils ont ouvert une nouvelle page de l'histoire de France. "Patience! Avec le temps, l'herbe devient du lait", dit la sagesse chinoise.

\section{Notes}

1. Voir Yahia Belaskri et Elisabeth Lesne (dir.), Algéries 50, Paris, Magellan \& Cie/CNHI, 2012.

2. Il faut ici saluer Jean Déjeux, défricheur du roman maghrébin et donc algérien, prolixe chroniqueur des pages "livres" de la revue. Voir l'hommage de Charles Bonn dans Hommes \& Migrations, n’ 1171 de novembre 1993.

3. Philippe Dewitte, ancien rédacteur en chef de Hommes \& Migrations, in "1950-2000, des Cahiers nord-africains à Hommes \& Migrations" (www.hommes-et-migrations.fr/index.php?id=5278).

4. Pascal Blanchard, Éric Deroo, Driss El Yazami, Pierre Fournier, Gilles Manceron (dir.), Le Paris arabe, Paris, La Découverte, 2003.

5. Dans son roman L'Enfant du peuple ancien (Pauvert, 2000), Anouar Benmaleck raconte l'histoire de Kader et Lislei, tous deux déportés en Nouvelle-Calédonie. Elle n'est pas une "communeuse" mais la victime de la répression des Versaillais; lui a bien participé à la révolte d'el Mokrani.

6. Voir, dans ce numéro, le texte de Laura Reeck, p. 122.

7. Gérard Noiriel, Immigration, antisémitisme et racisme en France (XIX'-XXe siècles). Discours publics, humiliations privées, Paris, Fayard, 2007, pp. 524-525.

8. Ibid., p. 567.

9. HCI, La France sait-elle encore intégrer les immigrés ?, Paris, La Documentation française, 2011.

10. Voir la chronique signée par André Videau dans Hommes \& Migrations, n ${ }^{\circ} 1293$, septembre-octobre 2011.

11. "Les FTP algériens et le sauvetage d'enfants juifs", in El Watan, le 16 mai 2005.

12. Voir Noureddine Elkarati, La Constitution de la population algérienne du département de Seine-Saint-Denis (1921-1999). Étude de géographie historique, Lille, Ed. ANRT, Thèse à la carte, 2006.

13. Tassadit Imache, “Écrire tranquille?”, in Esprit, décembre 2001.

14. Dixit Sophie Verney-Caillat sur : www.rue89.com/planete89/2010/07/24/rabhi-cest-la-civilisation-la-plusfragile-de-lhistoire-159675. Voir aussi le tout récent : Pierre Rabhi, La Part du colibri, La Tour d'Aigues, L'Aube, 2011. 15. Alain Tarrius, La Mondialisation par le bas. Les nouveaux nomades de l'économie souterraine, Paris, Balland, 2005. 